\title{
INVESTIGACIONES GEOGRÁFICAS
}

INSTITUTO UNIVERSITARIO DE GEOGRAFÍA

UNIVERSIDAD DE ALICANTE

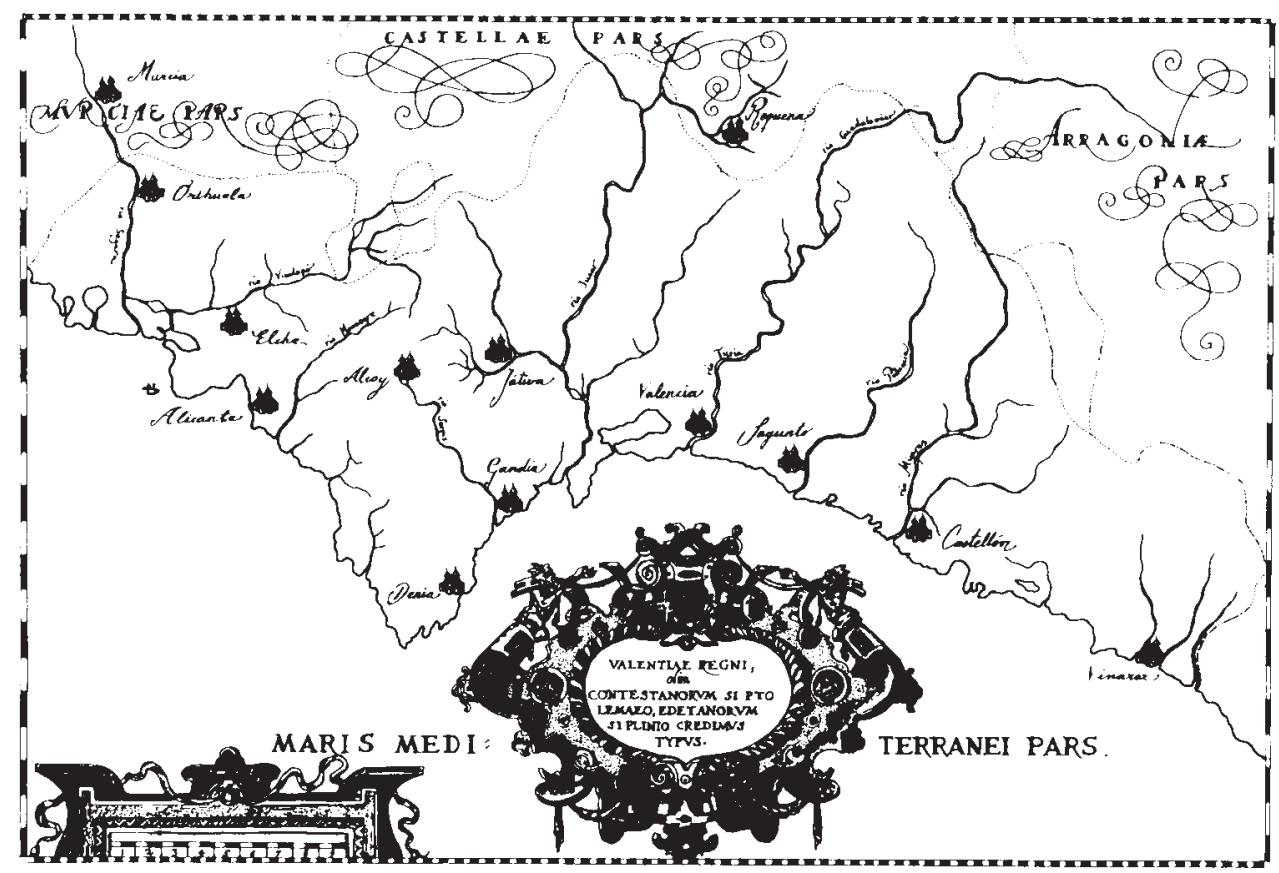

ANALES DE LA UNIVERSIDAD DE ALICANTE INSTITUTO UNIVERSITARIO DE GEOGRAFÍA ENERO - JUNIO 1999

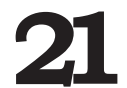




\title{
EVOLUCIÓN EN EL ESTUDIO DEL FENÓMENO ENSO (EL NIÑO-OSCILACIÓN DEL SUR): DE ANOMALÍA «LOCAL» A LA PREDICCIÓN DE VARIACIONES CLIMÁTICAS GLOBALES
}

\author{
Óscar Marcos Valiente*
}

\section{RESUMÉ}

Evolution dans l'étude du phénomène ENSO (El Niño-Oscillation du Sud): d'anomalie «locale» a la prediction des variations climatiques globales.

Cet article présente l'evolution historique dans l'étude du phénomène ENSO. Cette evenement, qui seulement semblait présenter repercusions dans la region littorale de Perou et Equador, est considéré aujourd'hui comment un des plus solides parametres pour développer predictions climatiques à court terme. Le concept qui a possibilité cette changement c'est la teleconnection climatique, qui signale l'existence des rélations entre anomalies climatiques dans lieux éloignées. Après l'intens ENSO du 1982-83, qui produit sévères alterations dans les systemes oceanique et atmosferique du planète, et dommages economiques et humains a regions diverses, l'étude de cettes connections a augmentée. L'immense quantité d'études scientifiques réalisées pendant les dernières dix ans, a permis connaître les mécanismes phyisiques qui expliquent le développement des teleconnections.

Palabras clave: Anomalías Climáticas, Climatología, El Niño, ENSO, Oscillación del Sur, Predicción Climática, Teleconexiones.

\begin{abstract}
Evolution in the study of ENSO ( $\mathrm{El}$ Niño-Southern Oscillation) events: from a «local» anomaly to the prediction of global climate variations.

This paper presents a historic evolution of ENSO events related studies. Such event, which at first only seemed to produce reliable effects along Peru and Ecuador coastal
\end{abstract}

\footnotetext{
* Departament de Geografia Física i Anàlisi Geogràfica Regional de la Universitat de Barcelona. Este trabajo se ha realizado gracias a la aplicación de una beca pre-doctoral (FPI, MEC), y forma parte de una investigación dirigida por el Dr. José Luis Luzón Benedicto.
} 
regions, is now considered as one of the best parameters to develop short-term climatic predictions. The concept which has made this change possible is the climatic teleconnexion, which shows the existence of strong relationships between distant regions. After the very strong 1982-83 ENSO event, which produced serious anomalies on climatic and oceanic systems, as well as economic and human damages in several regions, the study of these teleconnexions was strengthened. The huge amount of scientific studies produced during the last ten years, has allowed to deepen in the knowledge of the physical mechanisms that explain the development of the teleconnexions.

\section{RESUM}

\section{Evolució en l'estudi del fenomen ENSO (El Niño-Oscilllació del Sud): d'anomalia «local» a la predicció de variacions climàtiques globals.}

L'article presenta l'evolució històrica en l'estudi del fenomen ENSO. Aquest event, que en principi només semblava produir repercusions destacables a la regió litoral de Perú i Equador, ha passat a ser considerat como un dels paràmetres més sòlids per realitzar prediccions climàtiques a curt termini. El concepte que ha possibilitat aquest canvi és el de teleconnexió climática, que indica l'existència de fortes relacions entre anomalies climàtiques a intrets allunyats entre sí. Després de l'intens ENSO de 1982-83, que produí greus alteracions en els sistemes oceànic i atmosfèric de tot el globus, acompanyades per danys econòmics i humans en diverses regions, es reforçà l'estudi d'aquestes connexions. La realització de gran quantitat d'estudis científics en aquesta línia durant els darrers deu anys, ha permès aprofundir en el coneixement dels mecanismes físics que expliquen el desenvolupament de les teleconnexions.

\section{Introducción}

El tema estrella dentro de los estudios de climatología durante los últimos años ha sido, sin ninguna duda, el cambio climático y la previsión de los efectos que se derivarían sobre la población. Los medios de comunicación han contribuido enormemente a esta tendencia, convirtiendo el cambio climático en una cuestión que, sin menospreciar su verdadera importancia, ha alcanzado una repercusión social posiblemente sobredimensionada en la que cualquier manifestación meteorológica extrema, tal como un período demasiado caluroso, una ola de frío fuera de época, una sequía, o una secuencia de precipitaciones que deriva en inundación, pasa a ser considerada inmediatamente como una manifestación inequívoca del cambio climático.

Buena parte de los estudios realizados por la comunidad científica internacional en relación al cambio climático tienen su razón de ser en la profundización del conocimiento al respecto de cómo las variaciones de temperatura, precipitación y otros parámetros climáticos, afectarían a la economía y la población de diversas regiones del planeta. No obstante, dichos análisis socioeconómicos bajo un panorama climático diferente al actual, resultado de predicciones numéricas, no puede pasar del nivel de la hipótesis, puesto que las alteraciones previstas pueden retrasarse, acelerarse, o incluso no producirse, en función de la intervención de múltiples factores que pueden modificar las previsiones inicialmente establecidas.

Existen, sin embargo, otros fenómenos climáticos recurrentes que sí permiten desarrollar un análisis efectivo sobre las consecuencias de una alteración climática en la sociedad, la economía y la población de determinados lugares, ya que se producen in situ y pueden ser estudiadas en toda su extensión. Estos eventos nos dan la posibilidad de considerar toda 
la diversidad de elementos que intervienen, tanto a nivel climático en su desarrollo, como a nivel socioeconómico al contemplar los efectos que producen sobre los habitantes de una región concreta y su economía. El lector podría pensar que hago referencia a diversos desastres naturales, tales como huracanes, inundaciones, sequías y otras manifestaciones climáticas, y en parte sería un planteamiento correcto, puesto que todas ellas nos han permitido conocer las dinámicas extremas de la naturaleza, y en base a ello se han desarrollado sistemas de previsión para minimizar los negativos efectos que producen sobre nuestras actividades económicas.

A pesar de todo, el estudio de esas manifestaciones por sí mismas no nos permite plantear la vertiente predictiva que tan atractivo hace el concepto de cambio climático. Esa perspectiva queda ampliamente satisfecha al analizar una manifestación climática también considerada como desastre natural, el fenómeno El Niño, desconocido por el público en general, y caracterizado por un calentamiento anómalo del Pacífico ecuatorial central y oriental, centrado especialmente en el litoral de Perú y Ecuador. Esta región latinoamericana, que habitualmente presenta unos bajos niveles pluviométricos por la subsidencia atmosférica predominante derivada de la presencia de aguas frías de la corriente de Humboldt, ve como la aparición de El Niño produce una serie de alteraciones sobre la dinámica oceánica que favorece el desarrollo de precipitaciones torrenciales y episodios de inundaciones con efectos catastróficos.

El fenómeno El Niño, como podrá comprobar el lector en este artículo, se ha convertido en los últimos quince años en una de las herramientas más válidas para hallar el factor causante o detonante de algunas alteraciones climáticas muy significativas a nivel planetario, tanto por su extensión como por su magnitud. Así pues, el objetivo del presente ensayo es mostrar como El Niño, que parecía tener un carácter exclusivamente local, presenta implicaciones globales, produciendo modificaciones en la dinámica de la atmósfera y de los océanos que se proyectan a lugares muy lejanos, manifestándose en cambios en los regímenes de precipitaciones, temperaturas o presión atmosférica, así como en alteraciones de los ecosistemas marinos y terrestres.

\section{El Niño, una alteración oceánica de incidencia local}

El fenómeno El Niño es conocido como tal desde hace más de cien años, al percibir los pescadores de las costas de Perú y Ecuador la aparición, con periodicidad anual, de una corriente de aguas cálidas procedente del norte en una región en la que habitualmente predominan las aguas frías. Como el fenómeno hace su aparición regularmente hacia las fechas navideñas, los pescadores de Paita (localidad litoral peruana), le otorgaron el nombre de El Niño, en honor a la festividad del Niño Jesús que esos días se celebra (Lockwood, 1984; Caviedes, 1984a; Yarnal, 1985; Ramage, 1986; Cucalon, 1987; Deser y Wallace, 1987; Enfield, 1989, 1992; Diaz y Kiladis, 1992).

La costa sudamericana del Pacífico oriental en estas latitudes presenta una superficie oceánica especialmente fría en comparación con el resto de las aguas ecuatoriales, debido a que los vientos predominantes del este (alisios), en conjunción con la fuerza de Coriolis, desplazan las aguas cálidas hacia el oeste, lo cual obliga a que las aguas más frías de niveles profundos afloren para sustituir la masa de agua perdida, y a que la presencia de la corriente de Humboldt, procedente de la región antártica, proporciona agua con temperaturas relativamente bajas en los niveles oceánicos subsuperficiales (Idyll, 1973).

Esta corriente cálida anual no tiene mayor incidencia sobre la región, por no alcanzar nunca latitudes más meridionales de los $2^{\circ} \mathrm{S}$ (Francou y Pizarro, 1985), pero ocasionalmente se intensifica, puesto que la debilitación de los alisios del Sudeste reduce la exten- 
sión del área de afloramiento de aguas frías, posibilitando que las aguas cálidas puedan alcanzar latitudes más australes de lo habitual. En condiciones normales, se observa una línea situada entre las islas Galápagos y el litoral ecuatoriano que separa la masa de agua cálida de la fría, conformando un frente oceánico con condiciones termométricas, de salinidad y de productividad biológica muy bien diferenciadas (Wooster y Reid, 1963; Cucalon, 1987; Domingo y De Miró, 1989); los cambios de intensidad de los vientos alisios fuerzan al frente a desplazarse hacia el sur.

En ocasiones, el pequeño debilitamiento anual de los alisios en el Pacífico oriental puede intensificarse. Cuando esto sucede, la ZCIT (Zona de Corvergencia Inter-Tropical) cruza el ecuador hasta situarse sobre los $5^{\circ} \mathrm{S}$, mientras que la temperatura superficial del mar continua ascendiendo en el Pacífico ecuatorial central y oriental, incluyendo el litoral de Perú y Ecuador, más allá de los meses habituales, desencadenando una serie de anomalías meteorológicas, oceánicas, biológicas y socioeconómicas sobre esta región litoral. Los estudios científicos (Caviedes, 1975, 1984a; Rasmusson y Carpenter, 1982; Ramage, 1986; Glantz, 1991; Trenberth, 1991; Enfield, 1992; Quinn y Neal, 1992) hablan de El Niño en referencia al fenómeno oceánico anómalo que produce las consecuencias ecológicas más graves sobre el ecosistema marino de la región, considerando el suave calentamiento anual del litoral como parte integrante de la dinámica oceánico-atmosférica habitual en la región.

\section{El Niño-Oscilación del Sur, interrelación de las dinámicas oceánica y atmosférica}

El estudio de El Niño no recibió demasiada atención, exceptuando a la propia comunidad científica peruana, hasta que Sir Gilbert Walker estudió la circulación atmosférica en el cinturón ecuatorial, lo que le permitió establecer, individualmente durante los años 20 (Walker, 1923), y conjuntamente con Bliss a lo largo de la década de los 30 (Walker y Bliss, 1930, 1932), el concepto de Oscilación del Sur.

La Oscilación del Sur (de aquí en adelante S.O., acrónimo inglés de Southern Oscillation), es un enlace de sistemas barométricos en el Océano Pacífico, visto como un balanceo de presiones entre el Pacífico tropical sudoriental y la región de Australasia, que controla las variaciones de presión superficial, vientos, temperatura y precipitaciones en los trópicos de esta cuenca oceánica (Horel y Wallace, 1981; Yarnal, 1985; Yarnal y Kiladis, 1985; Ramage, 1986; Deser y Wallace, 1987; Allan, 1988; Enfield, 1992; Diaz y Kiladis, 1992; Quinn y Neal, 1992).

Según esta relación barométrica, cuando las presiones aumentan en el anticiclón situado sobre el sudeste del Pacífico, tienden a descender en el sistema de bajas presiones de Indonesia y norte de Australia, y a la inversa (Horel y Wallace, 1981; Rasmusson y Carpenter, 1982, 1983; Lockwood, 1984; Ramage, 1986; Allan, 1988). Los dos sistemas de presiones quedan conectados con un flujo de aire desde el este hacia el oeste, que presenta intensidad variable en función de las diferencias barométricas existentes en cada momento (Yarnal, 1985; Ramage, 1986; Allan, 1988; Enfield, 1992; Diaz y Kiladis, 1992; Quinn y Neal, 1992).

La dinámica de El Niño y su relación con la circulación atmosférica en la cuenca pacífica empezó a desentrañarse gracias a los trabajos de Jacob Bjerknes, que en la década de los 60 descubrió los mecanismos físicos que relacionan el anómalo calentamiento del océano (principal manifestación de El Niño) con la S.O., y estableció una conexión directa entre los dos grandes sistemas que intervienen en este fenómeno climático: el oceánico y el atmosférico. Según Bjerknes (1966, 1969), el debilitamiento de los flujos del este en el Pacífico ecuatorial oriental, en conjunción con presiones anómalamente bajas en el Pacífi- 
co oriental y anómalamente altas en el Pacífico occidental, reduce el afloramiento de aguas frías y favorece un incremento de la temperatura superficial del mar (Horel y Wallace, 1981; Rasmusson y Carpenter, 1982, 1983; Yarnal, 1985; Yarnal y Kiladis, 1985; Deser y Wallace, 1987).

Es a partir de entonces cuando, dada la estrecha interrelación existente entre El Niño, como elemento oceánico, y la S.O., en su vertiente atmosférica, se empieza a hablar de El Niño-Oscilación del Sur, utilizando el acrónimo inglés ENSO (El Niño-Southern Oscillation). Este término, propuesto por Rasmusson y Carpenter (1982), ha sido plenamente aceptado por la comunidad científica, y su uso se ha generalizado para hablar del fenómeno climático anómalo con sentido global. En este artículo se utiliza el acrónimo inglés al hacer referencia a la Oscilación del Sur y al fenómeno ENSO, puesto que de esta manera concordamos con las siglas que utilizan los diversos autores a nivel internacional.

El incremento de temperatura en la cuenca pacífica, cuando tiene lugar simultáneamente al descenso barométrico y la debilitación del sistema de altas presiones en el sudeste del Pacífico, favorece la disminución de intensidad de los alisios, produciéndose así un bloqueo del afloramiento de aguas frías. El resultado es el ya referido calentamiento del litoral ecuato-peruano, y al desaparecer la estabilidad atmosférica generada por las aguas frías, la ZCIT no tiene obstáculo alguno para desplazarse hacia el sur, aproximándose al ecuador más allá de lo normal, hasta el punto de cruzarlo. En definitiva, las alteraciones oceánicas y atmosféricas, al incidir sobre la restructuración de la circulación atmosférica tropical, provocan modificaciones sobre la ZCIT que multiplican adicionalmente las alteraciones sobre el Pacífico ecuatorial oriental.

La presencia de masas de aire muy húmedas aportadas por la ZCIT, y de aguas muy cálidas a lo largo de todo el litoral de Ecuador y Perú actúan como fuente de combustible para la formación de células tormentosas, ya que el calor y el vapor de agua abundantes permiten el ascenso de masas de aire, la condensación, la formación de tormentas y la presencia de lluvias torrenciales (Idyll, 1973; Caviedes, 1975, 1984a; Rasmusson y Carpenter, 1982; Lockwood, 1984; Yarnal, 1985; Yarnal y Kiladis, 1985; Deser y Wallace, 1987; Aceituno, 1988; Jordán, 1991; Rasmusson, 1991; Diaz y Kiladis, 1992; Enfield, 1992; McGlone et al., 1992).

El mencionado desplazamiento de la ZCIT hacia el sur, que produce un aumento de actividad convectiva en el litoral ecuatorial de Sudamérica occidental, no sólo afecta a esta región, sino que se hace extensivo longitudinalmente hacia el oeste hasta los $165^{\circ} \mathrm{E}$ (Rasmusson y Carpenter, 1982), conformando un incremento de precipitaciones en todo el Pacífico ecuatorial central y oriental al sur del ecuador. De hecho, en el Pacífico central el incremento de temperaturas oceánicas, que ya se ha descrito como uno de los factores básicos para determinar la aparición de sectores de inestabilidad, es tan sólo ligeramente inferior al del Pacífico oriental (Rasmusson y Wallace, 1983; Caviedes, 1984a, b; Ardanuy et al., 1987; Lau y Sheu, 1991; Trenberth, 1991; Cole et al., 1992), pero el hecho de que las intensas precipitaciones y fuertes tormentas se desarrollen mayoritariamente sobre superficie oceánica, produce que las repercusiones sean mucho menos perceptibles por la población, a excepción de pequeños núcleos insulares escasamente poblados.

Las lluvias torrenciales del Pacífico central se hallan relacionadas en muchas ocasiones, además de con el calentamiento oceánico y el subsiguiente incremento de la convectividad, con una mayor frecuencia de formación de ciclones tropicales, que afectan a zonas normalmente ausentes de estos fenómenos, como las islas Hawaii o las Marquesas (Rasmusson y Carpenter, 1983; Enfield, 1992). El factor desencadenante es el desplazamiento del centro generador de ciclones desde el norte de Australia hacia longitudes más orientales, migración producida per los vientos anómalos del oeste que se observan durante el 
desarrollo de algunos episodios ENSO sobre la cuenca pacífica (Rasmusson y Wallace, 1983; Kiladis y Diaz, 1986).

El incremento de precipitaciones sobre el Pacífico oriental y central provoca una reorganización de la distribución pluviométrica en toda la franja ecuatorial, por lo que necesariamente tiene lugar una reducción de precipitaciones en alguna otra región del cinturón ecuatorial (Caviedes, 1984b; Diaz y Kiladis, 1992). Efectivamente, al oeste de los $180^{\circ}$ se produce un incremento barométrico que comporta la debilitación y migración hacia el nordeste del sistema de bajas presiones de Indonesia, lo que, sumado a la modificación en la circulación atmosférica tropical, produce un reajuste de la precipitación y la circulación de vientos en aquél sector del planeta.

Bajo condiciones ENSO, la zona de mayor actividad convectiva y liberación de calor latente, situada normalmente sobre Indonesia y el norte de Australia, no se desarrolla, sino que se desplaza hacia el Pacífico ecuatorial central, incidiendo en una reducción del ascenso de masas de aire sobre la región (Rasmussson y Carpenter, 1982; Krishnamurti et al., 1983; Ardanuy et al., 1987; Allan, 1988; Kuhnel, 1989; Suppiah, 1993). Este proceso,

\section{TABLA I}

Alteraciones oceánicas y atmosféricas generadas por el fenómeno ENSO en el Pacífico ecuatorial. Si el lector desea consultar los autores que han estudiado en profundidad cada una de estas alteraciones, puede acudir a Marcos (1996), donde aparece un exhaustivo listado bibliográfico específicamente dirigido a presentar los estudios que defienden las diversas modificaciones que el fenómeno ENSO pone en marcha en el Pacífico ecuatorial.

\begin{tabular}{|c|c|}
\hline Manifestación & Ámbito \\
\hline $\begin{array}{l}\text { Calentamiento de la temperatura superficial } \\
\text { del mar. }\end{array}$ & $\begin{array}{l}\text { Océano Pacífico ecuatorial central y costas occiden- } \\
\text { tales de América entre } 30^{\circ} \mathrm{N} \text { y } 30^{\circ} \mathrm{S} \text { (mayor incidencia } \\
\text { en el litoral de Perú). }\end{array}$ \\
\hline $\begin{array}{l}\text { Incremento de precipitación, tormentas tropicales } \\
\text { y inundaciones. }\end{array}$ & $\begin{array}{l}\text { Océano Pacífico ecuatorial central e islas de este sector } \\
\text { del Pacífico. }\end{array}$ \\
\hline $\begin{array}{l}\text { Entrada de aguas cálidas y aparición de especies } \\
\text { animales típicas del ámbito tropical. }\end{array}$ & Costa de Sudamérica, especialmente de Perú. \\
\hline Reducción en la concentración de nutrientes. & $\begin{array}{l}\text { Océano Pacífico ecuatorial y costa de América entre } \\
30^{\circ} \mathrm{N} \text { y } 30^{\circ} \mathrm{S} \text { (mayor incidencia en el litoral de Perú). }\end{array}$ \\
\hline Incremento de precipitación, inundaciones. & Costa del norte de Perú y sur de Ecuador. \\
\hline $\begin{array}{l}\text { Sequía, menor número de tormentas y ciclones } \\
\text { tropicales. }\end{array}$ & Indonesia, norte y este de Australia, y Melanesia. \\
\hline Incremento de temperaturas del aire. & Costa oeste de Sudamérica entre 5 y $35^{\circ}$ sur. \\
\hline $\begin{array}{l}\text { Disminución en la población de anchoveta y } \\
\text { otras especies autóctonas. }\end{array}$ & Costa de Perú. \\
\hline Reducción de precipitaciones. & Costa pacífica de América Central. \\
\hline Reducción en la población de aves marinas. & Costa peruana e islas del Pacífico ecuatorial. \\
\hline
\end{tabular}


al potenciar que en la región de Australasia predomine la subsidencia donde antes se producía convergencia, provoca una eventual disminución de precipitaciones que puede conducir a la aparición de sequías en los episodios extremos, hecho aceptado por numerosos autores. Evidentemente, el desplazamiento hacia el este de la actividad convectiva comporta un mayor número de tormentas y precipitaciones más abundantes sobre el Pacífico central, tal y como ya se indicó previamente.

El lector podrá encontrar una descripción más detallada del proceso de desarrollo de un fenómeno ENSO, así como de las alteraciones que se derivan sobre los sistemas oceánico y atmosférico, en las obras de diversos autores, entre los cuales puede destacarse a los siguientes: Wyrtki (1973, 1975), Rasmussson y Carpenter (1982), Barber y Chavez (1983), Cane (1983), Gill y Rasmusson (1983), McBride y Nicholls (1983), Philander (1983, 1985, 1990), Caviedes (1984a), Nicholls (1984a, b, c, 1992), Ramage (1986), Allan (1988), Glantz (1991), Jordán (1991), Trenberth (1991), Enfield (1992), Diaz y Kiladis (1992), Marcos (1996). En cualquier caso, para comprender la magnitud de las modificaciones que se generan sobre la cuenca Pacífica ecuatorial y tierras circundantes, en la Tabla I se presenta un listado de las más destacadas y reconocidas por la comunidad científica internacional.

\section{El fenómeno ENSO, alteraciones climáticas globales}

Una primera aproximación al estudio de las teleconexiones ya nos permitiría afirmar que el incremento de precipitaciones sobre el Pacífico central y oriental, característico de un ENSO, ha de comportar necesariamente una alteración pluviométrica en otro/s lugar/es del planeta, ya que el mecanismo atmosférico de autoequilibrio siempre tendrá tendencia a recompensar las variaciones.

Así pues, resulta necesario contrastar con mayor profundidad qué variaciones se producen en otros lugares del planeta en coincidencia con el fenómeno ENSO, mediante el estudio de los parámetros oceánicos y atmosféricos afectados: circulación atmosférica, temperaturas superficiales del mar, precipitaciones, temperatura del aire, sistemas ciclogenéticos y frontogenéticos, zonas de convergencia, etc. De esta forma, se hace posible la conexión de los episodios ENSO con períodos de clima anómalo en diversos lugares del planeta, lo cual permitirá poder realizar predicciones de cierta fiabilidad respecto a la posible aparición de eventos climáticos extremos (sequías, inundaciones, ciclones tropicales, etc.), y minimizar así los posibles efectos negativos que esos eventos podrían producir sobre la economía y la sociedad de las regiones afectadas.

A continuación se desarrollará una visión histórica del estudio de las teleconexiones, y se presentarán las múltiples relaciones climáticas a nivel planetario ligadas al fenómeno ENSO, aunque su análisis individualizado en profundidad será objeto de otros estudios.

\section{El estudio de anomalías climáticas en lugares lejanos}

Las anomalías hasta ahora analizadas en el Pacífico ecuatorial se proyectan más allá de su zona de influencia directa, generando variaciones climáticas relacionadas con el fenómeno ENSO en latitudes tropicales y latitudes medias (Bjerknes, 1966, 1969; Yarnal, 1985; Yarnal y Kiladis, 1985; Rasmusson y Wallace, 1983; Cole et al., 1992). Son las denominadas teleconexiones, definidas por Fleer (1981) como

"correlaciones en el tiempo y en el espacio de anormalidades a gran escala de la circulación atmosférica, determinadas estadística y empíricamente”. 
Se considera que la primera relación de anomalías climáticas a gran escala fue realizada por Sir Charles Todd, observador del gobierno del sur de Australia, publicada en ese mismo país durante la década de 1880, y en la que indicaba la coincidencia existente entre las sequías del sector septentrional de Australia y el debilitamiento del monzón en la India (Diaz y Kiladis, 1992; Nicholls, 1992). Evidentemente, en aquella época no se podía establecer una relación directa con el fenómeno ENSO, puesto que no se tenía el conocimiento sobre la dinámica de la S.O. y cómo este elemento atmosférico determinaba la aparición de sequías en Australia. No obstante, supuso el punto de partida para el desarrollo de los estudios de Hildebrandsson (1897), Lockyer y Lockyer (1902) y, especialmente, Walker y su colaborador (Walker, 1923; Walker y Bliss, 1930, 1932), que sí comenzaron a definir la importancia de las variaciones barométricas en el Pacífico y su incidencia sobre las alteraciones climáticas globales.

Walker halló algunas de las implicaciones de estas variaciones, documentando las características y el alcance de las oscilaciones barométricas y los cambios asociados de temperaturas y precipitaciones a nivel planetario. Así, tras haber definido la dinámica de la S.O., pudo comprobar que cuando las presiones se incrementaban en Australasia y simultáneamente se reducían en el sudeste del Pacífico, el monzón de verano sobre la India quedaba debilitado o no se desarrollaba, provocando eventuales sequías, en tanto que cuando la oscilación barométrica en el pacífico era inversa, el monzón se reforzaba, produciendo una pluviometría mucho más abundante (Walker, 1923). También definió algunas de las variaciones relacionadas con la S.O. durante el invierno del hemisferio norte, como la precipitación sobre las islas Hawaii, la temperatura en Canadá occidental o la presión a nivel del mar, temperaturas y precipitaciones en el sudeste de Estados Unidos.

Además del trabajo de Walker, en aquella misma época se desarrollaron otros estudios sobre variaciones de precipitación (Brooks y Braby, 1921; Leighly, 1933), siguiendo una línea de investigación que pretendía establecer una relación a gran escala de las diversas alteraciones climáticas que tenían lugar en la cuenca pacífica. Sin embargo, tras las aportaciones de Walker en los años 20 (Walker, 1923), y de Walker y Bliss en los años 30 (Walker y Bliss, 1930, 1932), se inicia un período de silencio durante el cual no se analizan nuevas asociaciones entre variaciones climáticas globales.

El interés renace un cuarto de siglo más tarde con los trabajos de Berlage (1957) a finales de los años 50 (en concreto, Berlage profundizó en el estudio de las relaciones entre la S.O. y las sequías en Indonesia), y de Troup (1961) y Bjerknes $(1966,1969)$ en los años 60 , que permitieron establecer la relación definitiva entre las variaciones de presión y el calentamiento del Pacífico oriental, incidiendo especialmente en el efecto que ejerce el incremento de temperatura superficial del mar sobre la circulación atmosférica ecuatorial (conocida con el nombre de circulación de Walker, en honor al científico que la estudió en profundidad), y sobre la circulación longitudinal norte-sur (que recibe el nombre de circulación de Hadley).

A partir de este momento comenzaron a proliferar los estudios centrados en hallar los mecanismos físicos que producen las interconexiones climáticas, dejando en un segundo plano el análisis puramente estadístico entre parámetros climáticos de lugares alejados entre sí, aunque sin llegar a prescindir totalmente de esa metodología (Rasmusson y Carpenter, 1982). Aquellos primeros estudios de correlaciones (entre los que se incluyen los de Walker, Walker y Bliss, Berlage o Troup), pese a conformar un estadio analítico insuficiente para conocer las verdaderas causas de la aparición de las teleconexiones, en su momento sí que permitieron identificar y definir los lugares concretos en los que se producían anomalías climáticas interrelacionadas, posibilitando la configuración de mapas de áreas afectadas (Yarnal, 1985). 
Pero el verdadero punto de inflexión para el reconocimiento de El Niño, no sólo como una manifestación local, sino también como una referencia esencial para establecer una relación climática global, fue el evento de 1972-73, que produjo graves pérdidas económicas en Perú y coincidió con alteraciones climáticas muy significativas en otros países, especialmente sobre Estados Unidos. Evidentemente, el cambio en la concepción se encuentra estrechamente relacionado con la aparición de anomalías climáticas ligadas al fenómeno ENSO sobre países desarrollados, puesto que hasta entonces sólo se interesaban por su estudio los científicos locales donde las manifestaciones eran más evidentes. A partir del momento en que se correlacionaron con el fenómeno ENSO las alteraciones climáticas observadas sobre Estados Unidos, Canadá y Europa occidental, el evento pacífico comenzó a recibir una especial atención. Los estudios dedicados al fenómeno El Niño fueron aumentando notablemente, aunque todavía en un número reducido, lo que permitió iniciar una progresiva profundización en el conocimiento de su dinámica.

La culminación de este proceso de incremento en la atención científica por el fenómeno pacífico alcanzó su máxima expresión a principios de los 80, coincidiendo con El Niño de 1982-83, que incluso recibió la atención de publicaciones dirigidas al público en general, como las estadounidenses Time o Newsweek (Caviedes, 1984b). A nivel científico, ese evento, considerado como el más intenso del siglo por las grandes alteraciones que provocó en los sistemas oceánico y atmosférico, supuso el inicio de un programa de estudio específico de diez años de duración auspiciado por la Organización Meteorólogica Mundial, y que recibió la denominación de programa TOGA (Tropical Ocean Global Atmosphere), es decir, Océano Tropical-Atmósfera Global. Su objetivo principal era analizar y comprender la incidencia que las variaciones en el océano tropical generan sobre la atmósfera global y el clima en diversos lugares del planeta.

Los estudios ligados a este programa tuvieron lugar entre 1985 y 1994, y permitieron conocer con bastante precisión la dinámica de desarrollo de un ENSO, constatando la variabilidad del propio fenómeno, que no siempre sigue un mismo patrón de evolución. Los mayores avances ligados al fenómeno ENSO han sido los encaminados a la creación de modelos de predicción climática a corto plazo (hacia seis meses de antelación); estos modelos, que acoplan los sistemas oceánico y atmosférico para simular con mayor precisión la incidencia que las alteraciones en el océano Pacífico producen sobre la atmósfera global, se han beneficiado del programa TOGA por el mayor conocimiento que dicho estudio ha producido de los diversos parámetros ligados al ENSO, es decir, vientos en superficie, temperatura superficial del mar, contenido de calor latente oceánico, corrientes oceánicas superficiales y subsuperficiales, nivel del mar, etc.

En base al desarrollo de esos modelos de predicción, que todavía se encuentran en proceso de validación, es posible conocer la variabilidad climática de diversos lugares del planeta, puesto que son las alteraciones generadas en la cuenca pacífica las que ponen en funcionamiento una dinámica climática anómala en todo el globo. En el siguiente apartado se podrá comprobar cuáles son las regiones específicas cuyo clima se halla estrechamente ligado a la dinámica oceánico-atmosférica del Pacífico, mediante las denominadas teleconexiones de anomalías climáticas.

\section{Teleconexiones del fenómeno ENSO}

En el anterior apartado se ha podido comprobar cómo el estudio sistemático de las anomalías climáticas planetarias ligadas al fenómeno ENSO ha tenido lugar tan sólo en los últimos veinte años. Las más significativas y más ampliamente reconocidas por parte de la 


\section{TABLA II}

Cuadro de manifestaciones climáticas y ecológicas a nivel planetario, relacionadas con la aparición de un fenómeno ENSO.

\begin{tabular}{|l|l|}
\hline \multicolumn{1}{|c|}{ Manifestación } & \multicolumn{1}{c|}{ Ámbito } \\
\hline Sequía. & Altiplano del sur de Perú y noroeste de Bolivia. \\
\hline $\begin{array}{l}\text { Incremento de precipitación, mayor número de } \\
\text { tormentas e inundaciones. }\end{array}$ & $\begin{array}{l}\text { Sur de Estados Unidos (sudoeste y sudeste) y Norte de } \\
\text { México (especialmente el noroeste). }\end{array}$ \\
\hline $\begin{array}{l}\text { Descenso de precipitación, sequía y menor número } \\
\text { de ciclones tropicales. }\end{array}$ & Caribe. \\
\hline Reducción de precipitaciones. & Amazonas brasileño. \\
\hline $\begin{array}{l}\text { Incremento de precipitación, aumento de tormentas } \\
\text { y mayor entrada de sistemas frontales. }\end{array}$ & Litoral central de Chile. \\
\hline $\begin{array}{l}\text { Reducción de precipitaciones y eventuales sequías } \\
\text { o Sêcas. }\end{array}$ & Nordeste de Brasil. \\
\hline Reducción de precipitaciones. & Venezuela y Colombia. \\
\hline Incremento de la temperatura superficial del mar. & $\begin{array}{l}\text { Altas latitudes del océano Pacífico oriental (costa del } \\
\text { noroeste de Estados Unidos, Canadá y Alaska). }\end{array}$ \\
\hline $\begin{array}{l}\text { Reducción de fauna marina autóctona (peces y } \\
\text { aves), y sustitución por especies de aguas cálidas. }\end{array}$ & Costa pacífica del nordeste de Estados Unidos y Canadá. \\
\hline Crisis de la industria pesquera del salmón. & Costa pacífica del nordeste de Estados Unidos. \\
\hline Reducción de precipitaciones invernales. & Islas Hawaii. \\
\hline Debilitamiento del monzón, con eventuales sequías. & India y sudeste de Asia. \\
\hline Incremento de precipitaciones. & $\begin{array}{l}\text { Extremo sur de la India, Sri Lanka y océano Índico } \\
\text { circundante. }\end{array}$ \\
\hline Incremento de precipitaciones. & Sur de Brasil y norte de Argentina. \\
\hline Aumento de temperaturas durante el invierno boreal. & Norte de Estados Unidos, sur de Canadá y Alaska. \\
\hline Incremento de precipitaciones. & Atlántico sudecuatorial, litoral de Guinea meridional. \\
\hline Fuerte sequía. & Sahel y Sahara occidentales. \\
\hline Descenso de precipitaciones, sequía. & Extremo sudeste de África y Madagascar. \\
\hline Reducción de precipitaciones. & Nordeste de China y sudeste de Mongolia. \\
\hline Reducción de precipitaciones y sequía. & $\begin{array}{l}\text { Meseta de Etiopía (extensible a Somalia y Yemen en } \\
\text { sequías severas). }\end{array}$ \\
\hline Ibérica) e Islas Británicas. \\
\hline
\end{tabular}




\section{PRINCIPALES TELECONEXIONES TERMOMÉTRICAS DEL \\ FENÓMENO E.N.S.O. SOBRE CONTINENTES Y OCÉANOS}

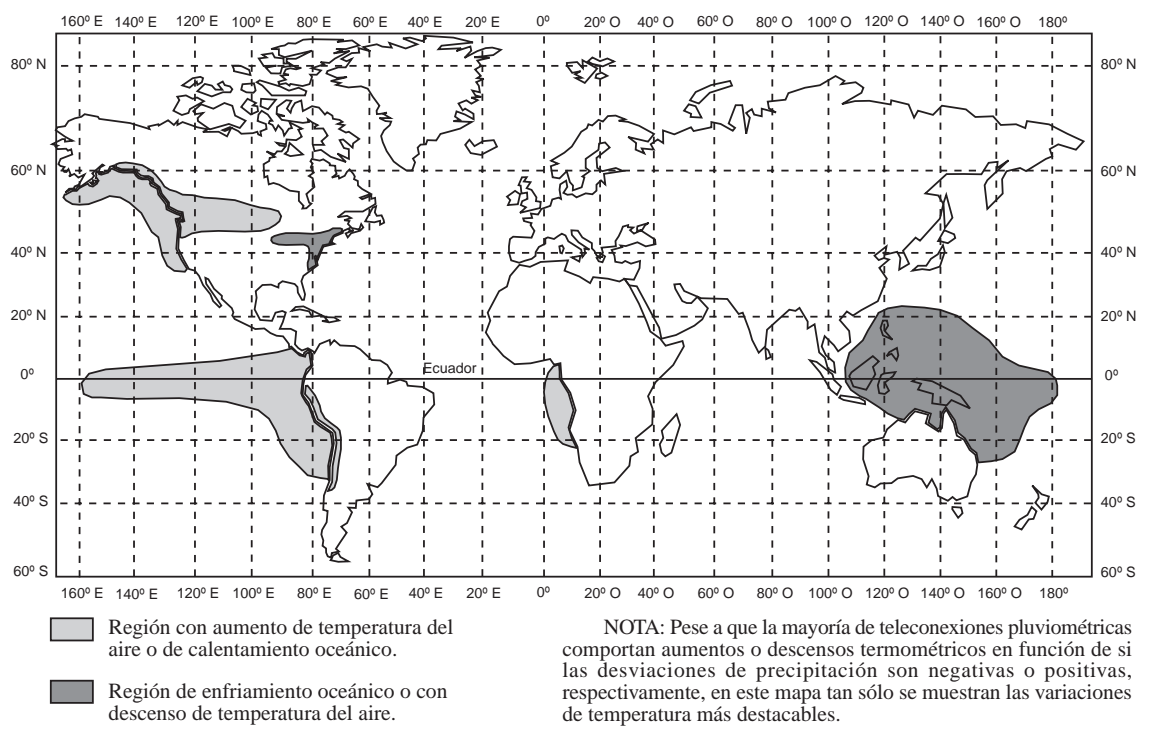

Figura 1. Distribución espacial de las teleconexiones termométricas sobre el planeta y de las alteraciones originales en la conca pacífica; se incluyen las variaciones más importantes de temperatura superficial del mar en regiones oceánicas, así como las desviaciones de temperatura atmosférica más destacadas sobre los continentes.

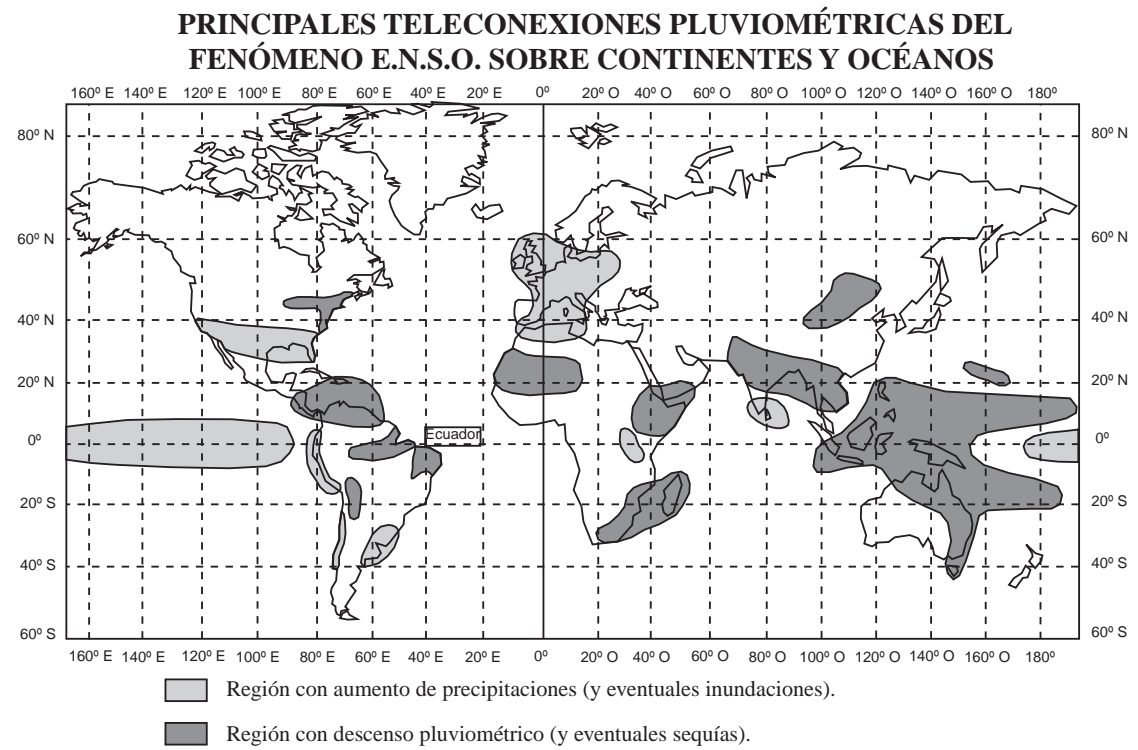

Figura 2. Distribución espacial de las teleconexiones pluviométricas sobre el planeta y de las alteraciones originales en la cuenca pacífica; se incluyen las variaciones más importantes de precipitación mostradas en la Tabla II, hecho que asegura su contrastación por parte de la comunidad científica. 
comunidad científica internacional aparecen reflejadas en la siguiente tabla, mientras que en los dos mapas adjuntos se muestra su distribución sobre el planeta.

Tal y como puede comprobarse por la distribución que se observa en los mapas de las figuras 1 y 2, las alteraciones que un fenómeno ENSO produce sobre la circulación en el Pacífico ecuatorial, con debilitación de la circulación este-oeste de Walker, no se limitan a las regiones circumpacíficas sino que, al producir una entrada adicional de energia en forma de calor latente hacia la circulación atmosférica de sentido meridional, la célula de circulación norte-sur de Hadley experimenta una notable modificación y queda reforzada en intensidad (Ardanuy et al., 1987; Rasmusson, 1991; Trenberth, 1991; Diaz y Kiladis, 1992; Enfield, 1992). El resultado es la proyección del calentamiento característico de El Niño hacia regiones remotas del globo, en especial hacia latitudes medias, en el proceso conocido como teleconexión de anomalías climáticas, concretadas en alteraciones pluviométricas, termométricas y barométricas en diversos puntos del planeta (Rasmusson y Wallace, 1983; Yarnal, 1985; Ropelewski y Halpert, 1987; Enfield, 1992; McGlone et al., 1992).

No obstante, estos cambios no se manifiestan de la misma forma ni en los mismos lugares en cada ENSO, puesto que en determinadas ocasiones El Niño no muestra actividad importante a nivel global (tal y como sucedió en 1917, 1923, 1932 ó 1943), mientras que en otros casos un fenómeno relativamente poco activo sobre el Pacífico oriental puede comportar una intensa actividad a escala planetaria, como los de 1904-1905 y 1918-1919 (Quinn et al., 1987; Quinn y Neal, 1992). La propia variabilidad del fenómeno ENSO provoca esta incertidumbre, que dificulta en cierta forma la total fiabilidad de los modelos de predicción anteriormente descritos. Hallar las causas de dicha variabilidad, junto al descubrimiento del factor desencadenante del inicio del fenómeno, son dos de los interrogantes más destacados que todavía deben ser esclarecidos para poder tener un conocimiento adecuado del funcionamiento de los eventos ENSO.

\section{Conclusiones}

La evolución histórica en el estudio del fenómeno ENSO a lo largo del siglo XX nos muestra como un evento que en principio tan sólo parecía presentar repercusiones a nivel local, es decir, limitadas a la región costera de Perú y Ecuador, ha pasado a ser considerado como uno de los parámetros más prometedores para realizar predicciones climáticas a corto plazo. Indudablemente, el desarrollo de los modelos de predicción basados en las anomalías oceánico-atmosféricas generadas en el Pacífico, todavía no ha alcanzado un grado de precisión suficiente como para garantizar su utilización, pero los resultados hasta ahora obtenidos permiten augurar que en un futuro ese objetivo sea alcanzado.

El concepto esencial que ha permitido la creación de modelos de predicción computerizados, son las teleconexiones, término inicialmente adoptado por Fleer a principios de los 80 para indicar la existencia de fuertes conexiones entre anomalías climáticas de lugares alejados entre sí. Partiendo de esa premisa, y tras el intensísimo evento ENSO de 1982-83, que produjo severas alteraciones en los sistemas oceánico y atmosférico de todo el globo, y que provocó severos daños económicos y humanos en regiones remotas, se inició el estudio en profundidad de dichas conexiones. La realización de innumerables estudios científicos, ha permitido profundizar en el conocimiento de los mecanismos físicos que conducen al desarrollo de las teleconexiones.

Hasta hace relativamente poco tiempo, los modelos climáticos comtemplaban exclusivamente el componente atmosférico (Modelos de Circulación General Atmosférica, 
MCGA), pero la experiencia acumulada con esos estudios ha aconsejado incorporar el sistema oceánico para obtener predicciones climáticas más ajustadas al verdadero comportamiento del clima, por lo cual a lo largo de los últimos cinco años han empezado a proliferar los Modelos Acoplados o Modelos de Circulación General Oceánico-Atmosférica (MCGOA). Parece inevitable pensar que el mejor conocimiento del fenómeno ENSO ha sido el principal factor explicativo del desarrollo de este último tipo de modelos.

En definitiva, las teleconexiones del fenómeno ENSO suponen, hoy en día, una de las bases más sólidas para la predicción de anomalías climáticas. Los estudios de cambio climático, mucho más conocidos socialmente, reciben una gran atención por parte de los medios de comunicación, que los divulgan de forma recurrente pese a tener un componente altamente especulativo. En cambio, existen otras posibilidades de establecer predicciones sobre situaciones climáticas futuras a corto plazo, basadas en experiencias y conocimientos sólidos, cuya repercusión social es mucho menor, pero con resultados científicamente más contundentes. Aunque el camino por recorrer es todavía largo, el avance que el estudio de las teneconexiones del fenómeno ENSO ha supuesto para los modelos de predicción climática, permite afirmar que la línea de investigación ligada a esta anomalía en el océano Pacífico es una de las más prometedoras y, al mismo tiempo, una de más sólidas en el panorama científico actual.

\section{Bibliografía}

ACEITUNO, P. (1988): «On the functioning of the Southern Oscillation in the South American sector. Part I: Surface Climate». Monthly Weather Review, 116, pp. 505-524.

ALLAN, R.J. (1988): «El Niño-Southern Oscillation influences in the Australasian region». Progress in Physical Geography, 12, pp. 313-348.

ARDANUY, P.E., CUDDAPAH, P. y LEE KYLE, H. (1987): «Remote Sensing of Water Vapor Convergence, Deep Convection, and Precipitation Over the Tropical Pacific Ocean During the 1982-1983 El Niño». Journal of Geophysical Research, 92, pp. 14204-14216.

BARBER, R.T. y CHAVEZ, F.P. (1983): «Biological consequences of El Niño». Science, 222, pp. 1203-1210.

BJERKNES, J. (1966): «A possible response of the atmospheric Hadley circulation to equatorial anomalies of ocean temperature». Tellus, 18, pp. 820-829.

BJERKNES, J. (1969): «Atmospheric teleconnections from the equatorial Pacific». Monthly Weather Review, 97, pp. 163-172.

BROOKS, C.E.P. y BRABY, H.W. (1921): «The clash of the trades in the Pacific». Quarterly Journal of the Royal Meteorological Society, 47, pp. 1-13.

CANE, M.A. (1983): «Oceanographic events during El Niño». Science, 222, pp. 1189-1194.

CAVIEDES, C.N. (1975): «El Niño 1972: its climatic, ecological, human, and economic implications». Geographical Review, vol. 65, pp. 493-509.

CAVIEDES, C.N. (1984a): «El Niño 1982-83». Geographical Review, 74, pp. 267-290.

CAVIEDES, C.N. (1984b): «Geography and the lessons from El Niño». The Professional Geographer, 36, pp. 428-436.

COLE, J.E., SHEN, G.T., FAIRBANKS, R.G. y MOORE, M. (1992): «Coral monitors of El Niño/ Southern Oscillation dynamics across the equatorial Pacific». DIAZ, H.F. y MARKGRAF, V. (1992): El Niño. Historical and Paleoclimatic Aspects of the Southern Oscillation. Cambridge University Press. Cambridge. (pp. 349-37).

CUCALON, E. (1987): «Oceanographic Variability off Ecuador Associated With an El Niño Event in 1982-1983». Journal of Geophysical Research, 92, pp. 14309-14322.

DESER, C. y WALLACE, M. (1987): «El Niño Events and Their Relation to the Southern Oscillation: 1925-1986». Journal of Geophysical Research, 92, pp. 14189-14196.

DIAZ, H.F. y KILADIS, G.N. (1992): «Atmospheric teleconnections associated with the extreme phases of the Southern Oscillation». DIAZ, H.F. y MARKGRAF, V. (1992): El Niño. Historical 
and Paleoclimatic Aspects of the Southern Oscillation. Cambridge University Press. Cambridge. (pp. 7-28).

DOMINGO, M. y DE MIRÓ, M. (1989): Breviario de oceanografía. Oikos-Tau (Col. Opera Geographica Minora). Barcelona. 194 pp.

ENFIELD, D.B. (1989): «El Niño, past and present». Reviews in Geophysics, 27, pp. 159-187.

ENFIELD, D.B. (1992): «Historical and prehistorical overwiew of El Niño/Southern Oscillation». DIAZ, H.F. y MARKGRAF, V. (1992): El Niño. Historical and Paleoclimatic Aspects of the Southern Oscillation. Cambridge University Press. Cambridge. (pp. 95-117).

FLEER, H.E. (1981): «Teleconnections of rainfall anomalies in tropics and subtropics». LIGHTHILL, J. y PEARCE, R.P. (eds.) (1981): Monsoon dynamics. Cambridge University Press. Cambridge. (pp. 5-18).

FRANCOU, B. y PIZARRO, L. (1985): «El Niño y la sequía en los Altos Andes Centrales: (Perú y Bolivia)». Bulletin de l'Institut Français d'Études Andines, 14, pp. 1-18.

GILL, A.E. y RASMUSSON, E.M. (1983): «The 1982-83 climate anomaly in the equatorial Pacific». Nature, 306, pp. 229-234.

GLANTZ, M.H. (1991): «Introduction». GLANTZ, M.H., KATZ, R.W. y NICHOLLS, N. (1991): Teleconnections Linking Worldwide Climate Anomalies. Cambridge University Press. Cambridge. (pp. 5-11).

HILDEBRANDSSON, H.H. (1897): «Quelques reserches sur les centres d'action de 1'atmosphere». Kon. Svenska Vetens.-Akad. Hand., 29, 33 pp.

HOREL, J.D. y WALLACE, J.M. (1981): «Planetary scale atmospheric phenomena associated with the Southern Oscillation». Monthly Weather Review, 109, pp. 813-829.

IDYLL, C. P. (1973): «La crisis de la anchoveta peruana». MOORE, J. R. (ed.) (1975): Oceanografía. H. Blume Ediciones. Madrid. (pp. 456-464).

JORDÁN, R. (1991): «Impact of ENSO events on the Southeastern Pacific region with special reference to the interaction of fishing and climate variability». GLANTZ, M.H., KATZ, R.W. y NICHOLLS, N. (1991): Teleconnections Linking Worldwide Climate Anomalies. Cambridge University Press. Cambridge. (pp. 401-430).

KILADIS, G.N. y DIAZ, H.F. (1986): «An analysis of the 1977-78 ENSO episode and comparison with 1982-83». Monthly Weather Review, 114, pp. 1035-1047.

KRISHNAMURTI, T.N., SURGI, N. y MONOBIANCO, J. (1983): «Recent results on divergent circulations over the global tropics». Papers of Meteorological Research, Journal of Meteorology of the Socialist Republic of China, 6, pp. 41-62.

KUHNEL, Y. (1989): «Spatial and temporal variations in Australo-Indonesian region cloudiness». International Journal of Climatology, 9, pp. 395-405.

LAU, K.-M. y SHEU, P.J. (1991): «Teleconnections in global rainfall anomalies: Seasonal to interdecadal time scales». GLANTZ, M.H., KATZ, R.W. y NICHOLLS, N. (1991): Teleconnections Linking Worldwide Climate Anomalies. Cambridge University Press. Cambridge. (pp. 227-256).

LEIGHLY, J.B. (1933): «Marquesan meteorology». University of California Publication in Meteorology, 6 ( $\left.\mathrm{n}^{\circ} 4\right)$, pp. 147-172.

LOCKYER, N. y LOCKYER, W.J.S. (1902): «On the similarity of the short-period pressure variation over large areas». Proceedings of the Royal Society of London, 73, pp. 457-470.

LOCKWOOD, J.G. (1984): «The Southern Oscillation and El Niño». Progress in Physical Geography, 8, pp. $102-110$.

MARCOS, O. (1996): Sequeres al Nordest de Brasil i el fenòmen El Nino. Aproximació a l'estudi de la seva interrelació. Tesina de licenciatura inédita. Departament de Geografia Física i A.G.R. Universitat de Barcelona. 150 págs.

McBRIDE, J.L. y NICHOLLS, N. (1983): «Seasonal relationships between Australian rainfall and the Southern Oscillation». Monthly Weather Review, 111, pp. 1998-2004.

McGLONE, M.S., KERSHAW, A.P. y MARKGRAF, V. (1992): «El Niño/Southern Oscillation climatic variability in Australasian and South America paleoenvironmental records». DIAZ, H.F. y MARKGRAF, V. (1992): El Niño. Historical and Paleoclimatic Aspects of the Southern Oscillation. Cambridge University Press. Cambridge. (pp. 435-462). 
NICHOLLS, N. (1984a): «El Niño Southern Oscillation and North Australian sea surface temperature». Tropical Ocean-Atmosphere Newsletter, 24, pp. 11-12.

NICHOLLS, N. (1984b): «The Southern Oscillation and Indonesian sea surface temperature». Monthly Weather Review, 112, pp. 424-432.

NICHOLLS, N. (1984c): «The Southern Oscillation, sea-surface temperature, and interannual fluctuations in Australian tropical cyclone activity». Journal of Climatology, 4, pp. 661-670.

NICHOLLS, N. (1991): «Teleconnections and health». GLANTZ, M.H., KATZ, R.W. y NICHOLLS, N. (1991): Teleconnections Linking Worldwide Climate Anomalies. Cambridge University Press. Cambridge. (pp. 493-510).

NICHOLLS, N. (1992): «Historical El Niño/Southern Oscillation variability in the Australasian region». DIAZ, H.F. y MARKGRAF, V. (1992): El Niño. Historical and Paleoclimatic Aspects of the Southern Oscillation. Cambridge University Press. Cambridge. (pp. 151-173).

PHILANDER, S.G.H. (1983): «El Niño Southern Oscillation Phenomena». Nature, 302, pp. $295-$ 301.

PHILANDER, S.G.H. (1985): «El Niño and La Niña». Journal of the Atmospheric Sciences, 42, pp. 2652-2662.

PHILANDER, S.G.H. (1990): El Niño, la Niña, and the Southern Oscillation. Academic Press. San Diego. 293 págs.

QUINN, W.H. y NEAL, V.T. (1992): «The historical record of El Niño events». BRADLEY, R.S. y JONES P.D. (eds.): Climate Since A.D. 1500. Routledge, Chapman and Hall. Londres. (pp. 623648).

QUINN, W.H., NEAL, V.T. y ANTÚNEZ DE MAYOLO, S.E. (1987): «El Niño over the past four and a half centuries». Journal of Geophysical Research, 92, pp. 14449-14461.

RAMAGE, C.S. (1986): «El Niño». VV.AA. (1986): El clima. Prensa Científica (Col. Libros de Investigación y ciencia). Barcelona. (pp. 67-75).

RASMUSSON, E.M. (1991): «Observational aspects of ENSO cycle teleconnections». GLANTZ, M.H., KATZ, R.W. i NICHOLLS, N. (1991): Teleconnections Linking Worldwide Climate Anomalies. Cambridge University Press. Cambridge. (pp. 309-343).

RASMUSSON, E.M. y CARPENTER, T.H. (1982): «Variations in tropical sea surface temperature and surface wind fields associated with the Southern Oscillation/El Niño». Monthly Weather Review, 110, pp. 354-384.

RASMUSSON, E.M. y CARPENTER, T.H. (1983): «The relationship between eastern equatorial Pacific sea surface temperatures and rainfall over India and Sri Lanka». Monthly Weather Review, 111, pp. 517-528.

RASMUSSON, E.M. y WALLACE, J.M. (1983): «Meteorological aspects of the El Niño/ Southern Oscillation». Science, 222, pp. 1.195-1.202.

ROPELEWSKI, C.F. y HALPERT, M.S. (1987): «Global and regional scale precipitation patterns associated with El Niño/southern Oscillation». Monthly Weather Review, 115, pp. 1.606-1.626.

SUPPIAH, R. (1993): «ENSO phenomenon and 30-50 day variability in the australian summer monsoon rainfall». Intenational Journal of Climatology, 13, pp. 837-851.

TRENBERTH, K.E. (1991): «General characteristics of El Niño-Southern Oscillation». GLANTZ, M.H., KATZ, R.W. y NICHOLLS, N. (1991): Teleconnections Linking Worldwide Climate Anomalies. Cambridge University Press. Cambridge. (pp. 13-42).

WALKER, G.T. (1923): «World weather I». Memoirs of the Indian Meteorological Department, 24, pp. 75-131.

WALKER, G.T. y BLISS, E.W. (1930): «World Weather IV. Some applications to seasonal foreshadowing». Memoirs of the Royal Meteorological Society, 3, pp. 81-94.

WALKER, G.T. y BLISS, E.W. (1932): «World Weather V». Memoirs of the Royal Meteorological Society, 4, pp. 53-84.

WOOSTER, W.S. y REID, J.L. Jr. (1963): «Eastern boundary currents». HILL, M.N. (ed.) (1963): The Sea. Interscience. New York. (pp. 253-280).

WYRTKI, K. (1973): «Teleconnections in the equatorial Pacific». Science, 180, pp. 66-68.

WYRTKI, K. (1975): «El Niño, the dynamic response of the equatorial Pacific Ocean to atmospheric forcing». Journal of Physical Oceanography, 5, pp. 572-584. 
YARNAL, B. (1985): «Extratropical teleconnections with El Niño/Southern Oscillation (ENSO) events». Progress in Physical Geography, 9, pp. 315-352.

YARNAL, B. y KILADIS, G. (1985): «Tropical teleconnections associated with El Niño/Southern Oscillation (ENSO) events». Progress in Physical Geography, 9, pp. 524-556. 